\title{
CD151 is associated with prostate cancer cell invasion and lymphangiogenesis in vivo
}

\author{
SUJITRA DETCHOKUL $^{1}$, BRADLEY NEWELL ${ }^{1,2}$, ELIZABETH D. WILLIAMS ${ }^{3-5}$ and ALBERT G. FRAUMAN ${ }^{1}$ \\ ${ }^{1}$ Clinical Pharmacology and Therapeutics Unit, Department of Medicine (Austin Health/Northern Health), \\ The University of Melbourne, Heidelberg, VIC; ${ }^{2}$ Department of Surgery and Urology, University of Melbourne, \\ Austin Hospital, Heidelberg, VIC; ${ }^{3}$ Monash Institute of Medical Research, Monash University, Clayton, Melbourne, VIC; \\ ${ }^{4}$ Australian Prostate Cancer Research Centre-Queensland, Queensland University of Technology, Brisbane, QLD; \\ ${ }^{5}$ Department of Surgery, St. Vincent's Hospital, University of Melbourne, Fitzroy, VIC, Australia
}

Received August 14,2013; Accepted September 18, 2013

DOI: 10.3892/or.2013.2823

\begin{abstract}
CD151, a member of the tetraspanin family, is associated with regulation of migration of normal and tumour cells via cell surface microdomain formation. CD151 was found in our laboratory to have a prognostic value in prostate cancer and is a promoter of prostate cancer migration and invasion. These roles involve association with integrins on both cell-cell and cell-stroma levels. Furthermore, CD151 plays a role in endothelial cell motility. CD151 expression was examined in three commonly used prostate cancer cell lines. We investigated CD151 expression, angiogenesis (microvessel density; MVD) and lymphangiogenesis (lymphatic vessel density; LVD) in an orthotopic xenograft model of prostate cancer in matched tumours from primary and secondary sites. CD151 was found to be heterogeneously expressed across different prostate cancer cell lines and the levels of CD151 expression were significantly higher in the highly tumorigenic, androgen-insensitive cells PC-3 and DU-145 compared to the androgen-sensitive cell line $\mathrm{LNCaP}(\mathrm{P}<0.05)$. The majority of in vivo xenografts developed pelvic lymph node metastases. Importantly, primary tumours that developed metastasis had significantly higher CD151 expression and MVD compared to those which did not develop metastasis $(\mathrm{P}<0.05)$. We identi-
\end{abstract}

Correspondence to: Professor Albert G. Frauman, Clinical Pharmacology and Therapeutics Unit, Department of Medicine (Austin Health/Northern Health), The University of Melbourne Level 5, Lance Townsend Building, Studley Road, Heidelberg, 3084 VIC, Australia

E-mail: albertf@unimelb.edu.au

Abbreviations: $\mathrm{CD}$, cluster designation; ECM, extracellular matrix; TM4SF, transmembrane-4-superfamily; SCID, severe combined immunodeficiency; MVD, microvessel density; LVD, lymphatic vessel density; PIN, prostate intra-epithelial neoplasia

Key words: tetraspanins, cell invasion, metastasis, angiogenesis, lymphangiogenesis and prostate cancer fied, for the first time, that CD151 expression is associated with LVD in prostate cancer. These findings underscore the potential role of CD151 and angiogenesis in the metastatic potential of prostate cancer. CD151 has a prognostic value in this mouse model of prostate cancer and may play a role in lymphangiogenesis. CD151 is likely an important regulator of cancer cell communication with the surrounding microenvironment.

\section{Introduction}

Prostate cancer is known to be a chronic disease and better detection and prognostication allowing long-term management is needed to improve quality of life for patients. Different prostate cancer cell lines express different levels of cluster designation (CD) antigens on their cell surface. Cell lines such as LNCaP, PC3 and DU145 are derived from metastatic lesions and, thus, have metastatic patterns which can be predicted by their CD phenotypes (1). The pattern of expression of CD antigens, thus, potentially provides prognostic information $(2,3)$. Some of these CD molecules are transmembrane-4-superfamily (TM4SF) members or tetraspanins, a family of 33 members in mammals (4-6). The exact biochemical functions of tetraspanins are still unknown, however, they have been linked to various processes including signal transduction pathways, cellular activation, proliferation, motility, adhesion, tissue differentiation, angiogenesis, tumour progression and metastasis. Most tetraspanins become downregulated in metastatic tumours; however, the CD151 glycoprotein was identified by Testa et al (7) to be the first tetraspanin member to play a role as a promoter of metastasis.

Binding of CD151 to the laminin binding integrins $\alpha 3 \beta 1$, $\alpha 6 \beta 1, \alpha 6 \beta 4$ and $\alpha 7 \beta 1$ promotes extracellular matrix (ECM) remodelling and there is evidence of localisation of CD151 and $\alpha 3$ integrins at the intercellular junction between endothelial cell-tumour cells, leading to tumour cell transendothelial migration across secondary sites (8). CD151 is a point of motility regulation on both cell-cell and cell-stroma levels which involves control of integrin activation and distribution (9). The findings from our laboratory show that CD151 has a better prognostication value than traditional Gleason grading and can distinguish aggressive forms of prostate cancer (10), 
and that in vitro manipulation of CD151 leads to change in prostate cancer migration (11). It is predicted that heightened CD151-positivity in prostate cancer cells will lead to more progressive disease; we have explored this in a mouse model of prostate cancer in the present study.

\section{Materials and methods}

Cell lines and reagents. The human prostate cancer cell lines PC-3, LNCaP and DU145 were obtained from the American Type Culture Collection (ATCC, Manassas, VA, USA). Cell lines were maintained and propagated as monolayer cultures in RPMI-1640 medium (Invitrogen Life Technologies, Carlsbad, CA, USA) respectively, with $10 \%$ foetal bovine serum (FBS) (Invitrogen Life Technologies) and $100 \mathrm{U} / \mathrm{ml}$ penicillin and $100 \mu \mathrm{g} / \mathrm{ml}$ streptomycin (Invitrogen Life Technologies). Antibodies used are monoclonal mouse anti-human CD151 antibody, 11B1.G4 (IgG2a) a kind gift from Professor L. Ashman's laboratory (Newcastle University, NSW, Australia) $(12,13)$, monoclonal mouse anti-human $\beta$-tubulin antibody (Invitrogen Life Technologies), monoclonal rat anti-mouse CD31 antibody (MEC 13.3) (BD Pharmigen ${ }^{\mathrm{TM}}$; BD Biosciences, San Jose, CA, USA) and monoclonal rat anti-mouse LYVE-1 antibody (XB-13) (Santa Cruz Biotechnology, Dallas, TX, USA).

Total cellular protein isolation and western blotting. Modified radioimmunoprecipitation (RIPA) buffer was used to extract proteins from the cells. Medium was removed from cells and cells were washed twice with ice-cold PBS before addition of ice-cold RIPA buffer containing 1X Complete Mini EDTA-free protease inhibitor tablet (Roche Diagnostics, Castle Hill, NSW, Australia). Protein concentrations of the whole cell lysates were determined using the Bradford assay (14). Western blot analysis was used to determine CD151 expression. Proteins were separated by SDS-PAGE. Protein bands were then transferred to nitrocellulose paper and incubated with CD151 antibody (Ab) and peroxidase conjugated anti-mouse $\mathrm{Ab}$, respectively. Peroxidase linked anti-mouse antibody was purchased from Amersham ${ }^{\mathrm{TM}}$ (GE Healthcare Biosciences, Rydalmere, NSW, Australia). $\beta$-tubulin levels were used as a loading control. Protein bands were visualized after chemiluminescent reaction.

RNA isolation. RNA was isolated using the Rneasy Mini kit (Qiagen) according to the manufacturer's instructions. RNA quantity was assessed using a UV spectrophotometer at A260 nm and RNA quality was determined using the A $260 \mathrm{~nm} / \mathrm{A} 280 \mathrm{~nm}$ ratio.

Reverse-transcript PCR and quantitative PCR. RNA was extracted from prostate cancer cell lines, LNCaP, PC-3 and DU-145 as previously described. cDNA synthesis were performed using M-MuLV kit (Invitrogen Life Technologies). cDNA samples were then analysed using CD151 (Assay ID Hs00388381_m1) and 18S (Assay ID Hs99999901_s1) (as control) TaqMan Gene Expression assays (Applied Biosystems/Life Technologies). PCR amplification was performed in a $25 \mu \mathrm{l}$ final volume (total $54 \mathrm{ng}$ cDNA per reaction) using Applied Biosystems 7500 real-time PCR system. mRNA expression of CD151 was normalized in relation to the control 18S expression. Data are expressed as fold difference to $\mathrm{LNCaP}$ cell line.

In vivo mouse model of prostate cancer. A severe combined immunodeficiency (SCID) mouse model orthotopically implanted with the human prostate cancer cell line, PC-3 constitutively expressing a fluorescent marker dsRED was used (15). In total, 12 SCID mice were implanted intraprostatically with human PC-3-DsRed cells, as a model of prostate cancer metastasis. Cells were inoculated in $0.01 \mathrm{ml}$ PBS into the prostate $\left(1 \times 10^{6}\right.$ cells/mouse). Mice were sacrificed 7 weeks after tumour cell inoculation.

The extent of tumour spread was examined by in vivo fluorescent imaging. For regular weekly in vivo imaging, the mice under study were first denuded of all hair in the region of interest. They were then anaesthetised with a 1-5\% isofluorane/95\% oxygen air mixture and placed under the light for imaging. The emission from DsRed was measured using a Fuji Film LAS-3000 camera housed alongside an image intensifier and light source in a custom built machine. Primary tumour volume was calculated using a common ellipsoid estimation length $\mathrm{x}$ width ${ }^{2} \mathrm{x} 0.5$ as previously described (16). Upon harvesting, mice were anaesthetised and primary tumours and regional lymph nodes were removed, weighed and processed for histology and immunohistochemical analyses. Tissues were fixed in $10 \%$ formalin, followed by paraffin embedding and sectioning. Mouse organs (spleen, liver and kidneys) were also removed and weighed for examination of any organ damage.

Immunohistochemistry. For histological analysis of the xenograft mouse model, tissue sections $(5 \mu \mathrm{m})$ were cut from formalin-fixed, paraffin-embedded tumour specimens of the implanted prostate cancer specimens and neighbouring lymph nodes (if tumour was found). Routine haematoxylin and eosin (H\&E) staining was performed for each specimen for histological analysis. CD151, CD31 (angiogenesis marker) and LYVE-1 (lymphatic marker) protein expression in tissue sections were measured using Dako LSAB ${ }^{+}$kit (Dako). CD151, CD31 and LYVE-1 stainings were performed as previously described $(10,15,17,18)$. Ab used was anti-human CD151 Ab (4 $\mu \mathrm{g} / \mathrm{ml}$ working concentration), CD31 Ab (diluted 1:10) and LYVE-1 Ab (diluted 1:50). Negative control immunostaining using isotype control antibody substituted for each primary antibody was also performed. Immunostainings were visualized with 3,3'-diaminobenzidine (DAB; Dako Australia Pty, Ltd., Campbellfield, VIC, Australia) and sections were then counterstained with haematoxylin.

Quantification of endothelial and lymphatic vasculature. Staining quantification was performed using light microscopy. CD31 and LYVE-1 stainings were analysed on digitised colour images and evaluated by counting of positive staining vessels. An intratumoural microvessel and lymphatic vessel were defined as any brown-stained (DAB immunoperoxidase stain with anti-CD31 antigen and anti-LYVE-1 antigen, respectively) cell or cell cluster clearly separated from adjacent vessels (19). Tumour vascularity was measured as microvessel density (MVD) and lymphatic vessel density (LVD) in areas containing intense neovascularisation 
A

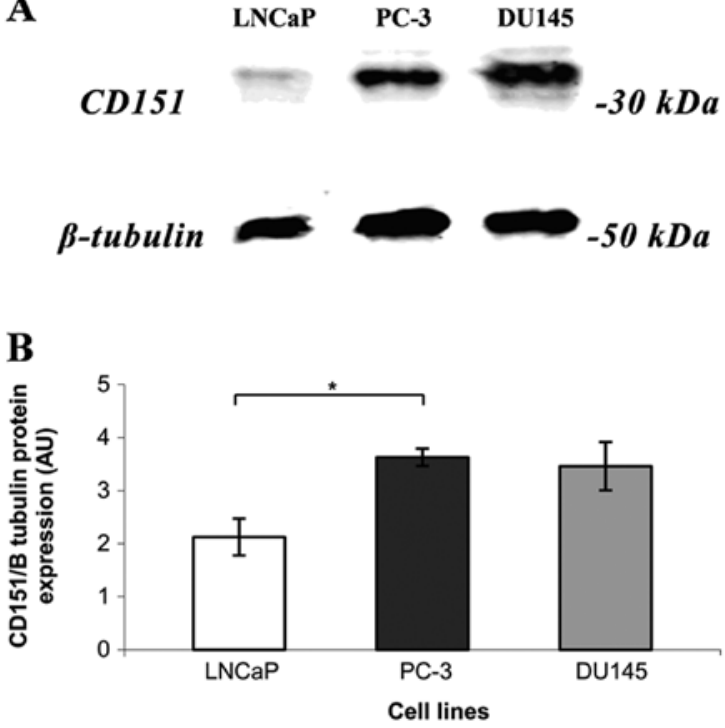

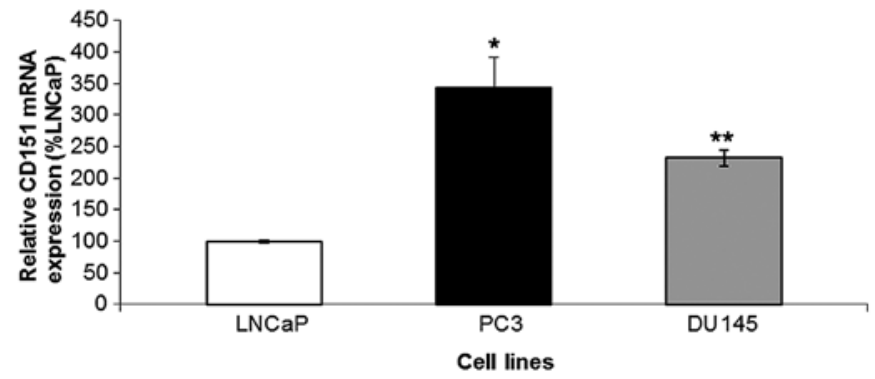

Figure 2. Relative CD151 mRNA expression in prostate cancer cell lines, LNCaP, PC-3 and DU145 cells. Relative expression was normalized to $18 \mathrm{~S}$. Student's t-test comparing to LNCaP cells to DU145 and PC-3 cells revealed 2-fold and 3-fold higher CD151 expression, respectively compared to LNCaP cells. ( $\mathrm{P}<0.05,{ }^{* *} \mathrm{P}<0.001 ; \mathrm{N}=3$; error bars, SEM).

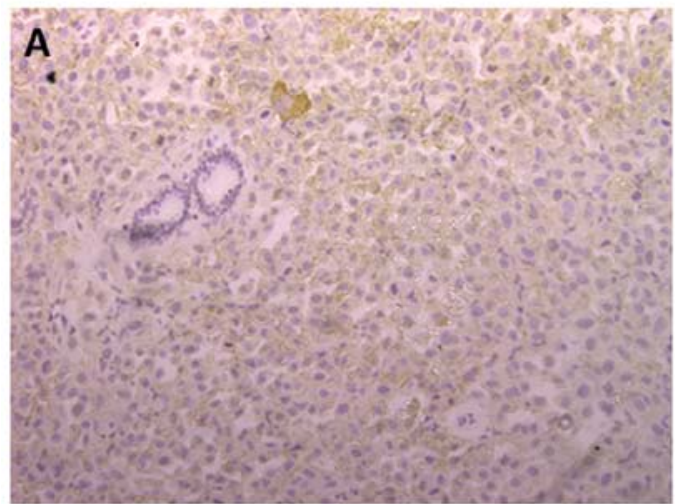

(neovascular 'hot spot'), as previously described $(19,20)$. The step-by-step procedures to identify these 'hot spots' were previously described (21).

Primary and secondary lesions were examined for MVD and LVD. Up to 10 hot spots (depending on tumour size) were selected at low magnification (x100) and vessels were counted in a representative high magnification (x200). The MVD or LVD ratios were calculated as vascular density $/\left(0.74 \mathrm{~mm}^{2}\right.$ for each x200 field). The vasculature ratios of primary tumour and secondary lymph node metastasis were compared and analysed. In addition, mice were also assigned into two groups of those that formed metastasis and those without metastasis. MVD and LVD of primary tumours of these two groups were also compared and analysed.

Statistical analysis. Statistical analysis was carried out using independent samples Student's t-tests. All P-values of $<0.05$ were considered to indicate a statistically significant result. Results are expressed as mean \pm standard error of the mean (SEM). The statistical tests performed for MVD and LVD were two-tailed, equal (F-value $>0.05$ ) or unequal variance (F-value $\leq 0.05$ ), Student's t-test. Pearson correlation was also performed to examine the relationship between CD151 staining, MVD, LVD and tumour weights.

\section{Results}

CD151 is expressed in commonly used prostate cancer cell lines. CD151 is heterogeneously expressed across all prostate cancer cell lines investigated. LNCaP, an androgen-responsive cell line, expressed low levels of CD151 protein and mRNA compared to two androgen-insensitive cell lines, PC-3 and DU-145 (Figs. 1 and 2, respectively).

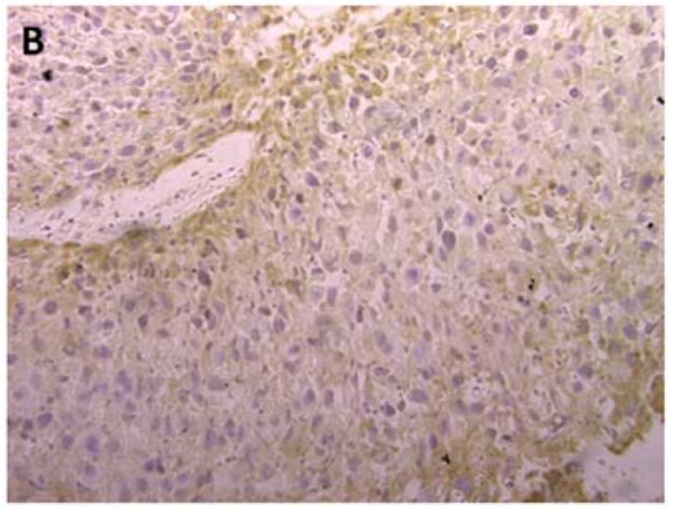

Figure 3. Representative areas of immunohistochemical CD151 tissue staining from (A) primary lesions, (B) secondary lymph node lesions (Original magnification, $\mathrm{x} 200$ ).

CD151 is a prognostic marker in mouse model of prostate cancer. All mice developed primary tumours at the site of inoculation (100\% tumour take rate) and 8 of $12(67 \%)$ mice developed pelvic lymph node metastases. CD151 expression was investigated via immunohistochemistry (Fig. 3). CD151 expression is predominantly localised in the cellular membrane and cytosol in prostate cancer cells. Weaker nuclear staining can also be seen. CD151 expression was examined in the primary and secondary lesions and no difference was seen between the two tumour sites (Fig. 4A). However, the expression of CD151 in primary tumour specimens from the group of mice forming metastasis was found to be significantly higher than in those mice without metastasis 

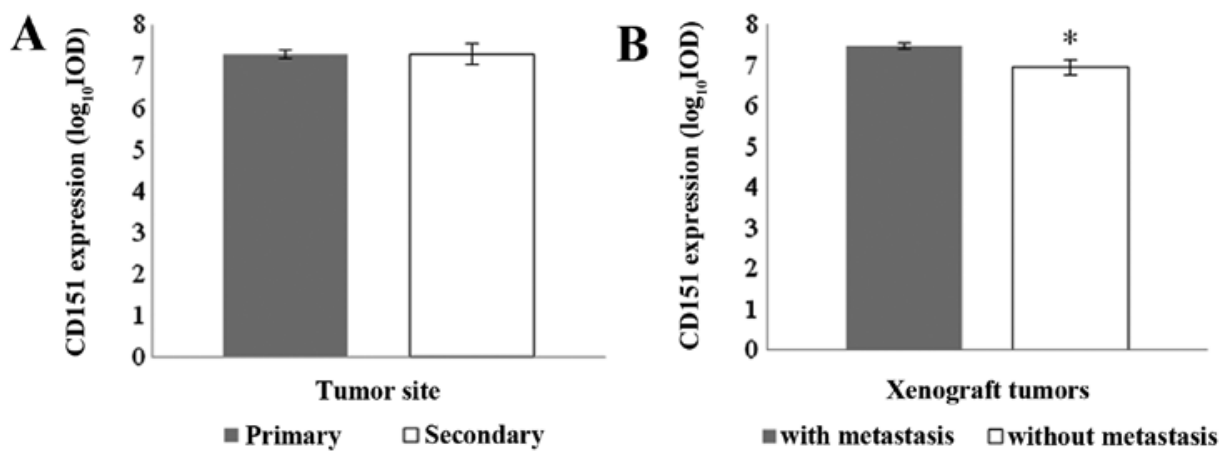

Figure 4. Primary lesions from mice that formed secondary metastases have higher CD151 expression compared to those that did not form metastasis (A) CD151 protein expression of primary xenograft tumours ( $\mathrm{N}=12$ ) and lymph node secondary metastases $(\mathrm{N}=8)$. Data presented as $\log _{10}$ integrated optical density (IOD). (B) CD151 protein expression (immunohistochemistry) in primary prostate xenograft with ( $\mathrm{N}=8$ ) and without (N=4) metastatic formation. Data shown as mean \pm SEM. ( $\mathrm{P}<0.05$; error bars, $\mathrm{SEM})$.
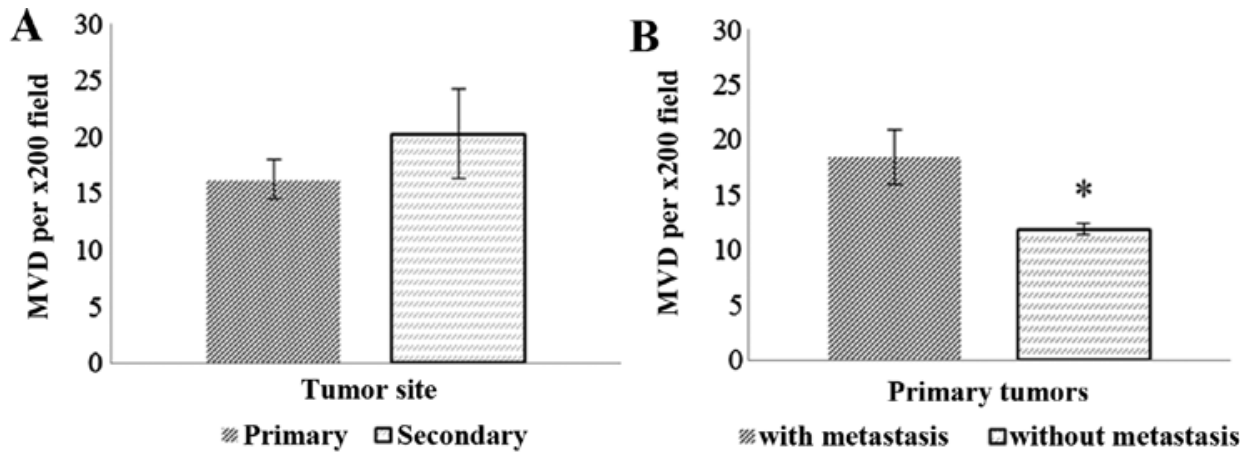

Figure 5. Angiogenesis is a prognostic marker in the PC-3 orthotopic xenograft mouse model of prostate cancer. (A) Mean MVD of primary xenograft tumours $(\mathrm{N}=12)$ and lymph node secondary metastases $(\mathrm{N}=8)$. (B) Mean MVD of primary prostate xenograft with $(\mathrm{N}=8)$ and without $(\mathrm{N}=4)$ metastatic formation. Data showing mean \pm SEM $\left({ }^{*} \mathrm{P}<0.05\right.$; error bars, $\left.\mathrm{SEM}\right)$.
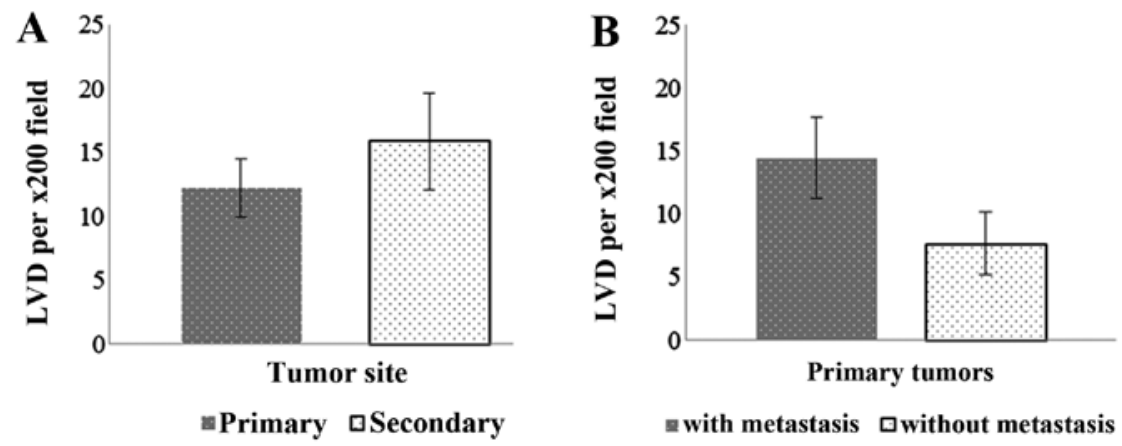

Figure 6. Lymphatic vessel density (LVD) in the PC-3 orthotopic xenograft model. (A) Mean LVD of primary xenograft tumours (N=12) and lymph node secondary metastases $(\mathrm{N}=8)$. (B) Mean MVD of primary prostate xenograft with $(\mathrm{N}=8)$ and without $(\mathrm{N}=4)$ metastatic formation. Data showing mean $\pm \mathrm{SEM}$.

formation (Fig. 4B). There was no evidence of non-specific staining in the control slides (data not shown). This confirms that CD151 has a prognostic value in this mouse model of prostate cancer.

Angiogenesis and lymphangiogenesis markers in mouse model of prostate cancer. We next investigated angiogenesis and lymphangiogenesis markers. The angiogenesis marker CD31 was used as a marker and microvessel density (MVD) and was measured in primary and secondary lesions. No difference in MVD was detected between primary and secondary lesions. However, MVD was significantly higher in primary prostatic xenografts forming metastases at nearby lymph nodes compared to primary lesions that did not form metastasis (Fig. 5). There was no association between tumour weight and MVD of the metastasis forming xenografts (data not shown).

Lymphatic vessel density (LVD) was not significantly different between primary and secondary lesions (Fig. 6) but there was a similar trend $(\mathrm{P}=0.19)$ toward increased LVD in primary xenografts forming metastases compared to those that did not form metastasis. 
Table I. Pearson correlation analysis of CD151, CD31 and LYVE-1 staining of primary PC-3 xenograft tumours.

\begin{tabular}{lcc}
\hline $\begin{array}{l}\text { Primary tissue } \\
\text { staining comparison }\end{array}$ & $\begin{array}{c}\text { Pearson correlation } \\
\text { (r-value) }\end{array}$ & $\begin{array}{c}\text { P-value } \\
\text { (two-tailed) }\end{array}$ \\
\hline CD151 vs. MVD & 0.48 & 0.058 \\
CD151 vs. LVD & 0.62 & $0.03^{\mathrm{a}}$ \\
MVD vs. LVD & 0.50 & 0.09 \\
\hline
\end{tabular}

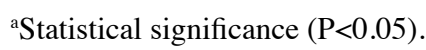

Table II. LVD in relation to metastasis formation in PC-3 orthotopic xenograft mouse model of prostate cancer.

\begin{tabular}{lcc}
\hline $\begin{array}{l}\text { LVD in } \\
\text { primary lesions }\end{array}$ & $\mathrm{n}$ & $\begin{array}{c}\text { Lymph node } \\
\text { metastasis }(\%)\end{array}$ \\
\hline High (> mean value of LVD) & 6 & $5(83)$ \\
Low (<mean value of LVD) & 6 & $3(50)$ \\
\hline
\end{tabular}

MVD and LVD relationships with CD151 expression levels and tumour growth. The level of CD151 expression, MVD and LVD was not significantly different between primary and secondary lesions. The relationships between CD151 staining, MVD and LVD were examined in primary lesions (Table I). CD151 expression was positively correlated with LVD (LYVE-1 staining) with statistical significance ( $\mathrm{r}=0.62$; $\mathrm{P}=0.03)$. The association between CD151 and MVD (CD31 staining) did not reach statistical significance $(r=0.48$; $\mathrm{P}=0.058$ ) and MVD and LVD were not correlated. MVD, LVD and CD151 expression did not correlate with tumour size (data not shown). Table II demonstrated that LVD may have a prognostic value; $83 \%$ of mice with high LVD progressed to form lymph node metastases.

\section{Discussion}

Tumour metastasis is a multi-factorial process and tumour cells are sensitive to local microenvironmental cues that regulate normal physiological functions such as wound healing and epithelial morphogenetic changes leading to malignant behaviour (22). CD151 is heterogeneously expressed across prostate cancer cell lines and the level of expression was noted to be higher in more highly aggressive cell lines. However, it has been shown that both $\mathrm{LNCaP}$ and PC-3 cell lines express integrin $\alpha 3 \beta 1$, which is a major partner of CD151 in the promotion of motility and metastasis (23). Integrin expression profiles between $\mathrm{LNCaP}$ and PC-3 cells differ, especially $\alpha 3$ integrin where LNCaPs have reduced expression compared to PC-3 cells which may explain the altered level of tumourigenicity between the two cell lines (24). This suggests the importance of integrins in invasive behaviour of prostate cancer. CD151- $\alpha 3 \beta 1$ association is found in most tissues that express $\alpha 3 \beta 1(25,26)$, which reinforces the importance of CD151 in the various functions of integrin $\alpha 3 \beta 1$. Our finding suggests that $\mathrm{CD} 151$ correlates with aggressiveness of the disease but whether CD151 has a role in transformation of androgen-responsive to androgen-irresponsive states cannot be concluded and is being explored in our laboratory.

While it is evident that CD151 expression correlates more with malignant types of prostate cancer, CD151 may have a role in angiogenesis as well as tumour motility and invasion. Our finding indicates that angiogenesis predicts tumour progression, which is in agreement with other studies (20,27-29). Although the exact mechanism is not known, there has been some evidence that suggests the role of CD151 in blood vessel formation in animal models. CD151 is expressed in vascular endothelial cells and associated with integrin $\beta 1$, $\beta 3, \beta 4, \alpha 2, \alpha 3, \alpha 5$ and $\alpha 6$ chains (30). A study using CD151null mice has shown a possible role of CD151 in tumour angiogenesis (31). These mice were viable and no phenotypic change was reported, however, pathological angiogenesis was greatly affected, supporting a pro-angiogenic role for CD151 specifically in pathological conditions $(31,32)$. Indeed, in animal models of myocardial ischaemia, introduction of CD151 via gene delivery improved capillary densities $(33,34)$. In vitro studies using endothelial cells have shown that CD151-integrin complexes have a role in endothelial cell proliferation, morphogenesis and migration, which are important in the angiogenesis process $(30,35,36)$. In prostate cancer, angiogenesis is associated with progression and prognosis (17,20,37-39).

CD151 involvement in angiogenesis has been demonstrated through the discovery of CD151-integrin complex localisation within endothelial cells and communication between tumour and endothelial cells via CD151, both essential for the formation of tumour neovascularisation $(8,36,40)$. Absence of CD151 in mouse lung endothelial cells led to alterations in angiogenic ability which was found to be through disruption in association with laminin-binding integrins and CD151-null mice also had defective pathological angiogenesis (31). Importantly, CD151 may have a role in regulation of tumour cell-endothelial cell dynamics and migrating behaviour of the endothelial cell which in turn promotes angiogenesis (40). In order to investigate the possible link between CD151 and angiogenesis, the changes in an angiogenesis marker were observed in a mouse model of prostate cancer. In prostate cancer, angiogenesis (using CD31 as a marker) was found to be an independent prognostic predictor (17). When angiogenesis was examined in the initiation and progression of prostate cancer using the spontaneous autochthonous transgenic adenocarcinoma of the mouse prostate (TRAMP) mouse model, CD31 staining revealed changes in angiogenesis patterns from low-grade prostate intra-epithelial neoplasia (PIN), high-grade PIN and amongst different prostate cancer grades (27). Our results indicate that CD151 expression and angiogenesis was a prognostic indicator in the PC-3 orthotopic xenograft mouse model and this is not correlated to tumour size.

The role of CD151 in angiogenesis is perhaps through assisting communication between tumour cells and endothelial cells (40). Many studies have found that CD151-integrin (especially $\alpha 3 \beta 1$ and $\alpha 6 \beta 4$ ) complexes are localised at the tumour cell and endothelial cell contact area $(8,36,40)$. Expression of CD151 is required for integrin distribution within the endothelial intercellular contacts, which in turn promotes angiogenesis (31). While cross-talk between tumour cells and endothelial cells assisting in transendothelial migration of 
tumour cells is important for angiogenesis, CD151 has been found to be a membrane linker, through which other signalling proteins stimulate the important regulator of endothelial cell function, endothelial nitric oxide synthase (41). Direct CD151 gene delivery into endothelial cells results in increased proliferation, migration and tube formation (42). Integrins, especially $\alpha 3 \beta 1$, have been found to be involved in the induction of angiogenesis by endothelial cells (43-46). Based on this information, it is likely that CD151 promotes pathological angiogenesis, via its association with integrin $\alpha 3 \beta 1$ and this association could be important at tumour or endothelial cells. Thus, inhibition of this complex via anti-CD151 agents might disrupt angiogenesis formation at either tumour or endothelial cells.

Our findings indicated statistically positive relationships between CD151 and LVD. Although LVD was not found to have prognostic value in this mouse model, the CD151 and LVD correlation suggest a possible role of CD151 in lymphangiogenesis in prostate cancer. This is particularly relevant in the PC-3 orthotopic xenograft model as the lymph nodes are the most common sites of metastases (15). This provides a basis for further exploration.

In conclusion, the involvement of CD151 in prostate cancer migration and metastasis is underscored and demonstrated in the present study. Because cell motility and invasion are decisive events for tumour metastasis, inhibiting CD151 is an attractive approach for inhibition of motility and invasion signalling pathway in prostate cancer. The present study supports further exploration into the role of CD151 in prostate cancer angiogenesis and lymphangiogenesis.

\section{Acknowledgements}

The present study was supported by grant funding to S.D. and A.G.F. from the Austin Medical Research Foundation and to E.D.W. from the National Health \& Medical Research Council of Australia and the Victorian Government's Operational Infrastructure Support Program. We thank Professor Leonie K. Ashman for supplying CD151 antibody for our studies.

\section{References}

1. Liu AY, Roudier MP and True LD: Heterogeneity in primary and metastatic prostate cancer as defined by cell surface $C D$ profile. Am J Pathol 165: 1543-1556, 2004.

2. Liu AY and True LD: Characterization of prostate cell types by CD cell surface molecules. Am J Pathol 160: 37-43, 2002.

3. Liu AY: Differential expression of cell surface molecules in prostate cancer cells. Cancer Res 60: 3429-3434, 2000.

4. Hemler ME: Tetraspanin functions and associated microdomains. Nat Rev Mol Cell Biol 6: 801-811, 2005.

5. Berditchevski F: Complexes of tetraspanins with integrins: more than meets the eye. J Cell Sci 114: 4143-4151, 2001.

6. Levy S and Shoham T: Protein-protein interactions in the tetraspanin web. Physiology 20: 218-224, 2005.

7. Testa JE, Brooks PC, Lin JM and Quigley JP: Eukaryotic expression cloning with an antimetastatic monoclonal antibody identifies tetraspanin (PETA-3/CD151) as an effector of human tumor cell migration and metastasis. Cancer Res 59: 3812-3820, 1999.

8. Longo N, Yanez-Mo M, Mittelbrunn M, et al: Regulatory role of tetraspanin CD9 in tumor-endothelial cell interaction during transendothelial invasion of melanoma cells. Blood 98 3717-3726, 2001.

9. Nishiuchi R, Sanzen N, Nada S, et al: Potentiation of the ligandbinding activity of integrin $\alpha 3 \beta 1$ via association with tetraspanin CD151. Proc Natl Acad Sci USA 102: 1939-1944, 2005.
10. Ang J, Lijovic M, Ashman LK, Kan K and Frauman AG: CD151 protein expression predicts the clinical outcome of low-grade primary prostate cancer better than histologic grading: a new prognostic indicator? Cancer Epidemiol Biomarkers Prev 13: $1717-1721,2004$

11. Ang J, Fang BL, Ashman LK and Frauman AG: The migration and invasion of human prostate cancer cell lines involves CD151 expression. Oncol Rep 24: 1593-1597, 2010.

12. Sincock PM, Mayrhofer G and Ashman LK: Localization of the transmembrane 4 superfamily (TM4SF) member PETA-3 (CD151) in normal human tissues: comparison with CD9, CD63 and $\alpha 5 \beta 1$ integrins. J Histochem Cytochem 45: 515-525, 1997.

13. Geary SM, Cambareri AC, Sincock PM, Fitter S and Ashman LK: Differential tissue expression of epitopes of the tetraspanin CD151 recognised by monoclonal antibodies. Tissue Antigens 58: 141-153, 2001.

14. Bradford MM: Rapid and sensitive method for quantitation of microgram quantities of protein utilizing principle of protein-dye binding. Anal Biochem 72: 248-254, 1976.

15. Zeng Y, Opeskin K, Goad J and Williams ED: Tumor-induced activation of lymphatic endothelial cells via vascular endothelial growth factor receptor-2 is critical for prostate cancer lymphatic metastasis. Cancer Res 66: 9566-9575, 2006.

16. Tomayko MM and Reynolds CP: Determination of subcutaneous tumor size in a athymic (nude) mice. Cancer Chemother Pharmacol 24: 148-154, 1989.

17. Mehta R, Kyshtoobayeva A, Kurosaki T, et al: Independent association of angiogenesis index with outcome in prostate cancer. Clin Cancer Res 7: 81-88, 2001.

18. Wroel T, Mazur G, Dziegiel P, et al: Density of intranodal lymphatics and VEGF-C expression in B-cell lymphoma and reactive lymph nodes. Folia Histochem Cytobiol 44: 43-47, 2006.

19. Weidner N, Semple JP, Welch WR and Folkman J: Tumor angiogenesis and metastasis - correlation in invasive breast carcinoma. N Engl J Med 324: 1-8, 1991.

20. Weidner N, Carroll PR, Flax J, Blumenfeld W and Folkman J: Tumor angiogenesis correlates with metastasis in invasive prostate carcinoma. Am J Pathol 143: 401-409, 1993.

21. Weidner N: Current pathologic methods for measuring intratumoral microvessel density within breast carcinoma and other solid tumors. Breast Cancer Res Treat 36: 169-180, 1995.

22. Quaranta V: Motility cues in the tumor microenvironment Differentiation 70: 590-598, 2002.

23. Schmelz M, Cress AE, Scott KM, et al: Different phenotypes in human prostate cancer: $\alpha 6$ or $\alpha 3$ integrin in cell-extracellular adhesion sites. Neoplasia 4: 243-254, 2002.

24. Witkowski CM, Rabinovitz I, Nagle RB, Affinito KS and Cress AE: Characterization of integrin subunits, cellular adhesion and tumorgenicity of 4 human prostate cell-lines. J Cancer Res Clin Oncol 119: 637-644, 1993.

25. Yauch RL, Kazarov AR, Desai B, Lee RT and Hemler ME: Direct extracellular contact between integrin $\alpha_{3} \beta_{1}$ and TM4SF protein CD151. J Biol Chem 275: 9230-9238, 2000.

26. Kazarov AR, Yang X, Stipp CS, Sehgal B and Hemler ME: An extracellular site on tetraspanin CD151 determines $\alpha 3$ and $\alpha 6$ integrin-dependent cellular morphology. J Cell Biol 158: 1299-1309, 2002.

27. Huss WJ, Hanrahan CF, Barrios RJ, Simons JW and Greenberg NM: Angiogenesis and prostate cancer: identification of a molecular progression switch. Cancer Res 61: 2736-2743, 2001.

28. Steiner I, Jung K, Miller K, Stephan C and Erbersdobler A: Expression of endothelial factors in prostate cancer: A possible role of caveolin-1 for tumour progression. Oncol Rep 27: 389-395, 2012.

29. Gray DR, Huss WJ, Yau JM, et al: Short-term human prostate primary xenografts: an in vivo model of human prostate cancer vasculature and angiogenesis. Cancer Res 64: 1712-1721, 2004.

30. Sincock PM, Fitter S, Parton RG, Berndt MC, Gamble JR and Ashman LK: PETA-3/CD151, a member of the transmembrane 4 superfamily, is localised to the plasma membrane and endocytic system of endothelial cells, associates with multiple integrins and modulates cell function. J Cell Sci 112: 833-844, 1999.

31. Takeda Y, Kazarov AR, Butterfield CE, et al: Deletion of tetraspanin CD151 results in decreased pathological angiogenesis in vivo and in vitro. Blood 109: 1524-1532, 2007.

32. Wright MD, Geary SM, Fitter S, et al: Characterization of mice lacking the tetraspanin superfamily member CD151. Mol Cell Biol 24: 5978-5988, 2004. 
33. Zuo HJ, Liu ZX, Liu XC, et al: Assessment of myocardial blood perfusion improved by CD151 in a pig myocardial infarction model. Acta Pharmacol Sin 30: 70-77, 2009.

34. Lan RF, Liu ZX, Liu XC, Song YE and Wang DW: CD151 promotes neovascularization and improves blood perfusion in a rat hind-limb ischemia model. J Endovasc Ther 12: 469-478, 2005.

35. Zhang XA, Kazarov AR, Yang X, Bontrager AL, Stipp CS and Hemler ME: Function of the tetraspanin CD151- $\alpha 6 \beta 1$ integrin complex during cellular morphogenesis. Mol Biol Cell 13: 1-11, 2002.

36. Yáñez-Mó M, Alfranca A, Cabanas C, et al: Regulation of endothelial cell motility by complexes of tetraspan molecules CD81/TAPA-1 and CD151/PETA-3 with $\alpha 3 \beta 1$ integrin localized at endothelial lateral junctions. J Cell Biol 141: 791-804, 1998.

37. Offersen BV, Borre M and Overgaard J: Immunohistochemical determination of tumor angiogenesis measured by the maximal microvessel density in human prostate cancer. APMIS 106: 463-469, 1998

38. Trojan L, Thomas D, Knoll T, Grobholz R, Alken P and Michel MS: Expression of pro-angiogenic growth factors VEGF, EGF and bFGF and their topographical relation to neovascularisation in prostate cancer. Urol Res 32: 97-103, 2004.

39. Zhu W and Dahut WL: Tumor angiogenesis as an early marker of long-term prostate cancer mortality. Future Oncol 6: 341-345, 2010.
40. Sadej R, Romanska H, Baldwin G, et al: CD151 regulates tumorigenesis by modulating the communication between tumor cells and endothelium. Mol Cancer Res 7: 787-798, 2009.

41. Zheng ZZ and Liu ZX: CD151 gene delivery increases eNOS activity and induces ECV304 migration, proliferation and tube formation. Acta Pharmacol Sin 28: 66-72, 2007.

42. Zuo HJ, Lin JY, Liu ZY, et al: Activation of the ERK signaling pathway is involved in CD151-induced angiogenic effects on the formation of CD151-integrin complexes. Acta Pharmacol Sin 31: 805-812, 2010.

43. Mitchell K, Svenson KB, Longmate WM, et al: Suppression of integrin $\alpha 3 \beta 1$ in breast cancer cells reduces cyclooxygenase- 2 gene expression and inhibits tumorigenesis, invasion, and crosstalk to endothelial cells. Cancer Res 70: 6359-6367, 2010.

44. Wang X, Ferreira AM, Shao Q, Laird DW and Sandig M: $\beta 3$ integrins facilitate matrix interactions during transendothelial migration of PC3 prostate tumor cells. Prostate 63: 65-80, 2005.

45. Mitchell K, Szekeres C, Milano V, et al: $\alpha 3 \beta 1$ integrin in epidermis promotes wound angiogenesis and keratinocyte-toendothelial-cell crosstalk through the induction of MRP3. J Cell Sci 122: 1778-1787, 2009.

46. Dominguez-Jimenez C, Yanez-Mo M, Carreira A, et al: Involvement of $\alpha 3$ integrin/tetraspanins complexes in the angiogenic response induced by angiotensin II. FASEB J 15: 1457-1459, 2001. 